\title{
Porous Hydrogels From Shark Skin Collagen Crosslinked Under Dense Carbon Dioxide Atmosphere
}

\author{
Susana Fernandes-Silva, Joana Moreira-Silva, Tiago H. Silva, \\ Ricardo I. Perez-Martin, Carmen G. Sotelo, João F. Mano, \\ Ana Rita C. Duarte, ${ }^{*}$ Rui L. Reis
}

The possibility to fabricate marine collagen porous structures crosslinked with genipin under high pressure carbon dioxide is investigated. Collagen from shark skin is used to prepare prescaffolds by freeze-drying. The poor stability of the structures and low mechanical properties require crosslinking of the structures. Under dense $\mathrm{CO}_{2}$ atmosphere, crosslinking of collagen pre-scaffolds is allowed for $16 \mathrm{~h}$. Additionally, the hydrogels are foamed and the scaffolds obtained present a highly porous structure. In vitro cell culture tests performed with a chondrocyte-like cell line show good cell adherence and proliferation, which is a strong indication of the potential of these scaffolds to be used in tissue cartilage tissue engineering.

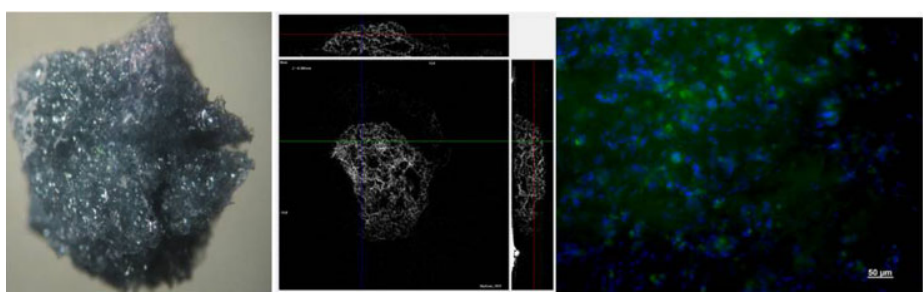

\section{Introduction}

Collagen is one of the most important and abundant proteins in human body, with 20 genetically distinct forms known until now. Generally, type I is the most studied for biomedical applications. ${ }^{[1]}$ The excellent biocompatibility and safety due to its inherent biological characteristics,

S. Fernandes-Silva, J. Moreira-Silva, T. H. Silva, J. F. Mano,

A. R. C. Duarte, R. L. Reis

3B's Research Group - Biomaterials, Biodegradables and

Biomimetics, University of Minho, Headquarters of the European

Institute of Excellence on Tissue Engineering and Regenerative

MedicineAvePark, 4806-9o9, Taipas, Guimarães, Portugal

E-mail: aduarte@dep.uminho.pt

ICVS/3B's - PT Government Associate LaboratoryBraga/

Guimarães, Portugal

R. I. Perez-Martin, C. G. Sotelo

CSIC, Inst Invest MarinasVigo, 36208, Galicia, Spain such as biodegradability and weak antigenicity, make collagen a primary resource in specific biomedical applications. ${ }^{[2]}$ Particularly interesting is the use of collagen for cartilage tissue engineering. In general, cartilage can be considered a hybrid material consisting of a collagen network together with other extracellular matrix components, mainly glycosaminoglycans (GAGs) such as chondroitin-4-sulfate and chondroitin-6-sulfate, present in the form of proteoglycans. ${ }^{[3,4]}$ Collagen is structurally formed as a triple helix by three extended protein chains that wrap around one another. Collagen and gelatin are different forms of the same macromolecule, however, gelatin is a partially hydrolyzed form of collagen. Heat denaturation easily converts collagen into gelatin. Collagen and gelatin are unique proteins. This uniqueness lays in the amino acid content, in particular non-polar amino acids such as Gly (30\%), Ala (10\%), and Pro (10\%)..$^{[5-7]}$ Despite the main industrial sources of collagen and gelatin being bovine and porcine skin, many studies have been conducted to extract 
collagen and gelatin from marine sources and have been used to screen their potential industrial applications. ${ }^{[5,6,8-10]}$ In marine environment, collagen can be found in different organisms and materials, ${ }^{[11,12]}$ such as marine sponges, ${ }^{[13]}$ jellyfish ${ }^{[14]}$ and fish skins, bones, cartilages and scales. ${ }^{[15-18]}$ The use of fish skin collagen and gelatin is expected to attract the interest of the industry as an alternative source, due to comparative unpopularity of porcine skin collagen and gelatin in relation to some religious reasons ${ }^{[19]}$ and mainly because there is an active discussion on the use of bovine derived collagen and gelatin due to the mad cow disease, bovine spongiform encephalopathy $(\mathrm{BSE})^{[20]}$ and the risk they pose in human. In contrast, fish collagen and gelatin have a relatively low risk of possessing unknown pathogens such as BSE. ${ }^{[21]}$

In this work, we propose the use collagen extracted from the shark Scyliorhinus canicula skin, as an alternative approach to overcome these drawbacks. The development of collagen structures able to promote cell adhesion and proliferation require crosslinking of the material, which is still a challenging task even though some reported have been described in the literature. Crosslinking of collagen is required to render stable structures in aqueous solution and different crosslinkers have been used, namely glutaraldehyde, $N$-(3-dimethylaminopropyl)- $N^{\prime}$ ethylcarbodiimide (EDC), $N$-hydroxysuccinimide (NHS), and genipin. Genipin is a natural crosslinking agent which possesses low toxicity and exhibits a characteristic dark blue color when reacting with amino groups in amino acids or proteins ${ }^{[22]}$ Crosslinking natural-based polymers with genipin has already proven its feasibility both under atmospheric conditions ${ }^{[23-26]}$ and under dense carbon dioxide atmosphere. ${ }^{[27]}$ It has also been reported that crosslinked collagen structures can be achieved with genipin after $48 \mathrm{~h}$ of reaction under atmospheric conditions. $^{[28]}$

The use of carbon dioxide as a reaction media has been reported for different purposes, namely, sub and supercritical fluid technology have proven to be successful in the preparation of crosslinked hydrogel structures. ${ }^{\text {[27,29-33] }}$ Carbon dioxide is the most commonly used solvent in supercritical fluid technology particularly due to its low toxicity, non-flammability, and mild critical point $\left(P_{\mathrm{C}}=73\right.$ bar and $\left.T_{\mathrm{C}}=31^{\circ} \mathrm{C}\right)$. Furthermore, $\mathrm{CO}_{2}$ is considered a GRAS solvent (Generally Regarded as Safe), ready available and inexpensive. For these reasons, it allows processing thermolabile compounds without compromising their bioactivity. ${ }^{[34]}$ Different techniques based on sub and supercritical fluids have been described in the literature and novel processes continue to be reported. One of the major advantages of these technologies is its versatility, which allows the production of porous structures from different materials. Recently, overcoming the processing difficulties faced with some natural polymers due to their high degree of crystallinity or hydrophobicity, the hydrogel foaming technique has been described. ${ }^{[35-37]}$ Polymers such as chitosan or elastin have been successfully processed and the in vitro biological performance reported demonstrated great potential for tissue engineering and regenerative medicine applications.In the present study, collagen prescaffolds were prepared using collagen extracted from skins of the shark specie $S$. canicula and further crosslinked with genipin under high-pressure $\mathrm{CO}_{2}$. The matrices obtained were characterized and their biological performance was assessed in vitro.

\section{Experimental Section}

\subsection{Collagen Isolation and Pre-Scaffold Preparation}

Fish skin was cut in small pieces, about $10 \mathrm{~g}$ of this material was weighed and mixed with $0.1 \mathrm{M} \mathrm{NaOH}$ and stirred in a cold room for 24h. After centrifugation, supernatant was removed and the residue was washed with distilled water (three times). Washed residue was stirred overnight with $0.5 \mathrm{M}$ acetic acid. After this acetic solubilization step, the extract was centrifuged at $10^{\circ} \mathrm{C}$ and $10000 \mathrm{~g}$ for $20 \mathrm{~min}$. Supernatant was purified through dialysis against distilled water for $2 \mathrm{~d}$. Dialyzed collagen extract was freezedried and stored frozen until further used.Pre-scaffolds were produced by dissolving the freeze-dried collagen in $0.5 \mathrm{M}$ acetic acid preparing solutions with different collagen concentrations 0.5 , 1 , and $2 \mathrm{wt} \%$. These solutions were stirred overnight, filtered, transferred for 96 -well microplates, frozen at $-80^{\circ} \mathrm{C}$ and freezedried.

\subsection{Sodium Dodecylsulfate/Polyacrylamide Gel Electrophoresis (SDS-PAGE)}

Samples for SDS-PAGE were prepared by adding $100 \mu \mathrm{L}$ of Laemmli sample buffer (1\% dithiothreitol was used instead of 2-ME) to $50 \mu \mathrm{g}$ of protein and heating for $4 \mathrm{~min}$ at $100{ }^{\circ} \mathrm{C}^{[38]} \mathrm{An}$ aliquot $(10 \mu \mathrm{L})$ of this mixture was applied to each well. Gels $\left(100 \times 750 \times 0.75 \mathrm{~mm}^{3}\right)$ were prepared according to the procedure of Laemmli (1970) and were subjected to electrophoresis at $15 \mathrm{~mA}$ using a Mini-Protean II Cell (Bio-Rad). Following electrophoresis, the gels were stained with 0.04\% Coomassie Blue in 25 vol\% ethanol and 8\% acetic acid for $30 \mathrm{~min}$ at $60^{\circ} \mathrm{C}$. Excess stain was removed with several washes of destaining solvent (ethanol 25 vol\%, acetic acid 8 vol\%.Molecular weight of subunits of ASC was estimated using molecular weight standards from Sigma-Aldrich (Molecular Weight Marker 30 000200000 SDS6H2) and analyzing the gel with the software Quantity One from Biorad.

\subsection{Micro-DSC}

Freeze-dried samples were solubilized in $0.050 \mathrm{M}$ acetic acid (about $5 \mathrm{mg}$ of freeze-dried sample per $\mathrm{mL}$ ). Thermostability of ASC solutions were measured in a microcalorimeter DSC III from Setaram (France). All samples were introduced in the calorimeter at $5{ }^{\circ} \mathrm{C}$ and left for $1 \mathrm{~h}$ to stabilize, after temperature increase was set to $1^{\circ} \mathrm{C} \mathrm{min}^{-1}$ up to $52{ }^{\circ} \mathrm{C}$. Two 
parameters were determined, namely, the maximum denaturation temperature and transition temperature by the onset method.

\subsection{Pre-Scaffold Crosslinking With Genipin by Dense $\mathrm{CO}_{2}$}

The structures were crosslinked with genipin under dense $\mathrm{CO}_{2}$ atmosphere. This technique promoted the crosslinking reaction inside a reactor at room temperature $\left(\mathrm{RT} \approx 24-25^{\circ} \mathrm{C}\right)$ and $50-55$ bar. Different crosslinking times were tested $(1,6$, and $16 \mathrm{~h})$ in order to evaluate which produced structures with better stability/crosslinking degree. Pre-scaffolds were immersed in different genipin solutions with concentrations of $0.1,0.3$, and $0.5 \mathrm{wt} \%$, respectively. Afterwards they were placed inside the high-pressure reactor and the reaction took place for the pre-determined period of time. After despressurization, scaffolds were removed from the reactor and were immediately immersed in $98 \%$ ethanol, to remove any residual genipin that might still be present. Afterwards, the 3D structures were frozen at $-80^{\circ} \mathrm{C}$ to stabilize the structure and were freeze-dried for $7 \mathrm{~d}$.

\subsection{Mechanical Properties - Compressions Tests}

Mechanical properties of scaffolds prepared was assessed by compression using an Instron 5540 (Instron Int. Ltd., High Wycombe, UK) universal testing machine with a load cell of 1 $\mathrm{kN}$. Compression testing was carried out at a crosshead of $2 \mathrm{~mm} \mathrm{~min}^{-1}$, until a maximum reduction in samples height of $60 \%$. The compressive modulus is defined as the initial linear modulus on the stress/strain curves and was determined as the average of at least three samples.

\subsection{Fourier-Transform Infrared (FTIR) Spectroscopy}

The infrared spectra of collagen structures were obtained with a Shimadzu- IR Prestige 21 spectrometer in the spectral region of $4000-800 \mathrm{~cm}^{-1}$ with resolution of $2 \mathrm{~cm}^{-1}$ at 32 scans. The samples were analyzed in the attenuated total reflectance mode.

\subsection{Determination of the Degree of Crosslinking - Ninhydrin Assay}

In order to calculate the crosslinking degree of the structures prepared $1.5 \mathrm{mg}$ of each scaffold was heated to $100^{\circ} \mathrm{C}$ in water bath with $1 \mathrm{~mL}$ ninhydrin solution ( 2 vol\% for $20 \mathrm{~min}$. The solution was cooled down to room temperature, and its optical absorbance was read in a microplate reader (Synergy HT, Bio-Tek Instruments) at $570 \mathrm{~nm}$. The amount of free amino groups in the control sample, pre-scaffold is proportional to the optical absorbance of the solution and was determined using a standard calibration curve of glycine. ${ }^{[24,25]}$

\subsection{Scanning Electron Microscopy (SEM)}

The morphology of the scaffolds prepared was observed by a Leica Cambridge S360 scanning electron microscope (SEM). The matrices were fixed by mutual conductive adhesive tape on aluminium stubs and covered with gold using a sputter coater. The same procedure was followed for the cells + scaffold construct after the cell seeding studies.

\subsection{Micro-Computed Tomography (Micro CT)}

The inner structure, porosity, and interconnectivity were evaluated by micro-computerized tomography using a Scanco 20 equipment (Scanco Medicals, Switzerland) with penetrative X-rays of $41 \mathrm{keV}$. The X-ray scans were acquired in high-resolution mode $(14.7 \mu \mathrm{m})$. CT Analyser ${ }^{\circledR}$ (SkyScan, Belgium) was used to visualize the 2D X-ray sections images of the scaffolds.

\subsection{In vitro Biological Performance}

\subsubsection{Cell Seeding}

In order to assess the scaffolds biological performance, after being prepared and sterilized through ethylene oxide, scaffolds were seeded with a chondrocyte cell line ATDC5 (ECACC, UK). Cells were cultured in Dulbecco's modified Eagle medium (DMEM)/nutrient mixture F-12 (DMEM/F12, Gibco, UK), supplemented with $2 \times 10^{-3}$ M L-Glutamine, $10 \%$ fetal bovine serum (FBS, Gibco, UK) and $1 \%$ antibiotic/antimycotic (Gibco, UK). Direct contact test was performed in which ATDC5 cells were seeded onto each scaffolds with a concentration of $5 \times 10^{5}$ cells $\mathrm{mL}^{-1}$ and cultured for 1,3 , and $7 \mathrm{~d}$.

\subsubsection{Cell Viability}

A 3-(4,5-dimethylthiazol-2-yl)-5-(3-carboxymethoxyphenyl)-2-(4sulfophenyl)-2H-tetrazolium (MTS) test was performed to assess the cellular viability after the pre-determined culture time. The reduction of this component to formazan is directly proportional to the cellular metabolism and is hereafter an indication of the cellular viability. The optical density (OD) of formazan was read at $490 \mathrm{~nm}$, on a multiwell microplate reader (Synergy HT, Bio-Tek Instruments). OD was determined for each time point and compared to polystyrene tissue culture plate, used as a positive control. All cytotoxicity screening tests were performed using three replicates.

\subsubsection{Cell Proliferation - DNA Quantification}

Cell proliferation was evaluated by quantifying the total amount of double-stranded DNA present in the cell/scaffold construct, at the different culture time points. Quantification was performed using the Quant-iTTM PicoGreen ${ }^{\circledR}$ dsDNA Assay Kit (Molecular Probes, Invitrogen, USA), after cell lysis by osmotic and thermal shock. The fluorescence was measured at an excitation wavelength of $485 / 20 \mathrm{~nm}$ and at an emission wavelength of 528/20 nm, in a microplate reader (Synergy HT). The DNA concentration for each sample was calculated using a standard calibration curve. Results were compared to the cell proliferation on the tissue culture polystyrene plate as means of assay control. Collagen scaffolds not seeded with ATDC-5 were used as sample control (data not shown). Assays were carried out in three replicates.

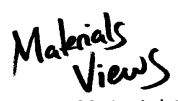

www.MaterialsViews.com
Macromol. Biosci. 2013, DOI: 10.1002/mabi.201300228

(C) 2013 WILEY-VCH Verlag GmbH \& Co. KGaA, Weinheim

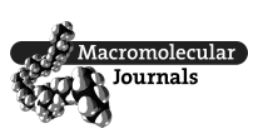




\subsection{Calcein-AM/4',6-Diamidino-2-phenylindole (DAPI) Staining}

The viability of cells and their proliferation in the cell/hydrogel constructs was assessed after calcein-AM/DAPI staining. After each time point constructs were incubated with a calcein-AM solution, prepared at a concentration of 1:1 000 in DMEM, for $10 \mathrm{~min}$. Constructs were then washed with phosphate-buffered saline (PBS), fixed in 10\% formalin (Sigma-Aldrich, Germany) for $15 \mathrm{~min}$ and washed again with PBS. Cell nuclei were stained with DAPI (Invitrogen, USA) and constructs were examined with an Axioplan Imager Z1M microscope (Zeiss, Germany), and images acquired and processed with AxioVision V.4.8 software (Zeiss, Germany).

\subsection{Statistical Analysis}

Statistical analysis of the data was performed using IBM SPSS Statistics Version 20. Normality was verified by the Shapiro-Wilk test. Normal distributed data were then analyzed using one-way ANOVA with Bonferroni's post test, when normality was not observed a non-parametric test, namely Kruskall-Wallis test was performed. Differences between the groups with $p<0.05$ were considered to be statistically significant.

\section{Results and Discussion}

Marine collagen has interesting inherent features, which confer it a high potential for the development of new materials for biomedical applications. However, the preparation of marine origin collagen matrices is still in its early stages of development and few papers have been reported in the literature. In this work, the development of porous hydrogels from shark skin collagen, extracted from S. canicula is evaluated.

Different extraction protocols have been established. The great variety of collagen sources and the differences in collagen type require the optimization of an extraction procedure for each specific raw material. ${ }^{[39,40]}$ Shark skin collagen was extracted following an already established protocol. ${ }^{[41]}$ In order to characterize the collagen obtained SDS-PAGE and micro-DSC techniques were employed. SDSPAGE analysis indicates that the extracted collagen from shark skin is mainly of type I, which is the one described to be the most suitable for biomedical applications. ${ }^{[42]}$ The collagen obtained was characterized by the presence of two alpha chains, alpha 1 of $126 \mathrm{kDa}$ and alpha 2 of $112 \mathrm{kDa}$, and a beta component of $210 \mathrm{kDa}$ (Figure 1), which resembles to the one of collagen type I of calf skin. Amino acid composition (Table 1) of the extracted collagen showed that Glycine content is highest among the amino acids present, since glycine is the every third residue amino acid (Gly-X-Y) in collagen molecules, except in the telopeptide regions. ${ }^{[4]}$ Positions $\mathrm{X}$ and $\mathrm{Y}$ of the repeated amino acid sequence of collagen are often occupied by hydroxyproline and proline, and the sum of both (imino acid content) has been described

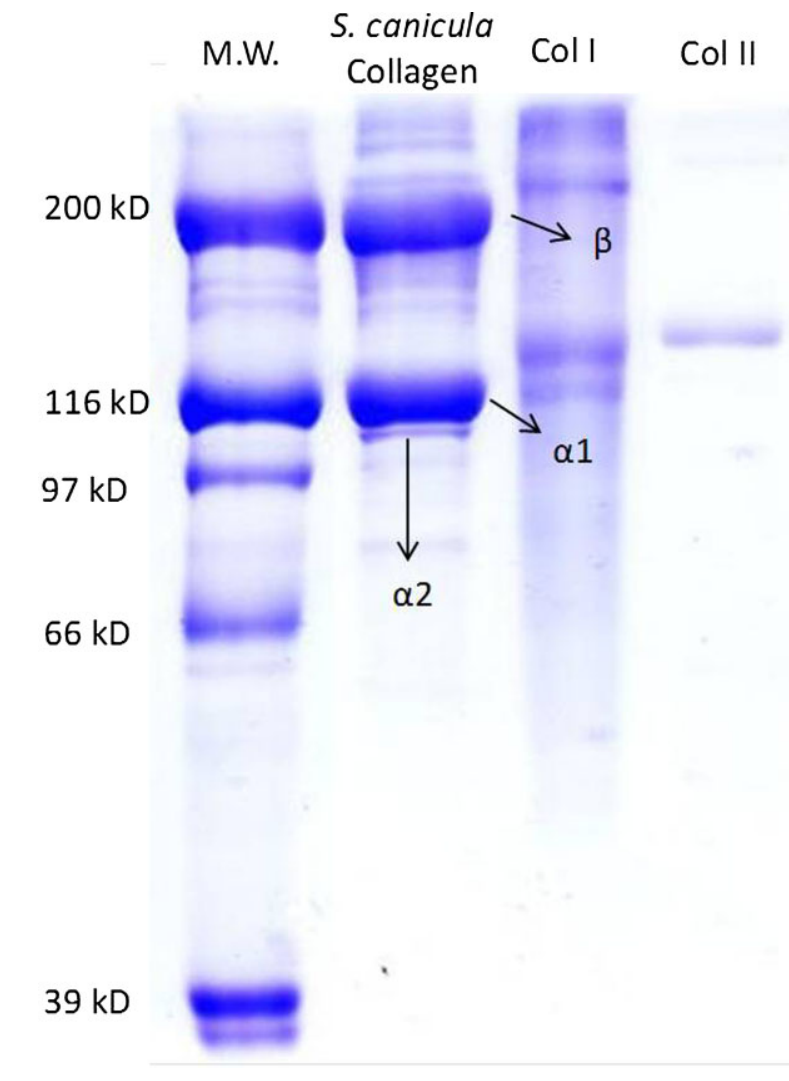

Figure 1. Representative SDS-PAGE blot showing S. canicula acid soluble collagen with the corresponding $\alpha 1, \alpha 2$, and $\beta$ subunits, calf skin collagen type 1 ( $\mathrm{Col} 1)$ and calf cartilage collagen type 2 (Col 2).

as responsible of some collagen properties. ${ }^{[4]}$ These values are similar to those presented by cold-water fish and lower than those of warm water fish. ${ }^{[45]}$ Calf skin shows a higher value of 215 imino acid residues/1000. Using micro-DSC analysis, a denaturation temperature of $33.15^{\circ} \mathrm{C}$ was observed.

\subsection{Crosslinking Reaction Under High-Pressure Carbon Dioxide}

In a first approach to prepare 3D porous structures, conventional freeze-drying was carried out. All scaffolds presented a cylindrical form according to the initial mold, a white color and spongy structure (Figure 2A). Due to the weak mechanical properties of such materials and low stability in different physiological solutions, crosslinking of the structures is essential in order to increase the stability of the architecture and tune its degradation rate according to the application envisaged. Genipin was chosen among the available crosslinking agents due to its natural origin and reported low toxicity. Nonetheless, genipin 
Table 1. Amino acid composition of S. canicula fish skin ASC and calf (residues/1000) $\times$ data from Zhang et al.[49]

\begin{tabular}{lrr} 
Amino acid & S. canicula & Calf \\
\hline Aalanine & 100 & 119 \\
Aarginine & 50 & 50 \\
Aaspartic a.cid/asparagine & 43 & 45 \\
Ccysteine & 1 & 0 \\
Gglutamic a.cid/glutamine & 68 & 75 \\
Gglycine & 338 & 330 \\
Hhistidine & 10 & 5 \\
Iisoleucine & 13 & 11 \\
Lleucine & 22 & 23 \\
Llysine & 27 & 26 \\
Hhydroxylysine & 6 & 7 \\
Mmethionine & 14 & 6 \\
Pphenylalanine & 15 & 3 \\
Hhydroxyproline & 87 & 94 \\
Pproline & 90 & 121 \\
Sserine & 59 & 33 \\
Tthreonine & 37 & 18 \\
Ttyrosine & 27 & 21 \\
Vvaline & 177 & 215 \\
Iimino acids & $49 \%$ & $44 \%$ \\
\% of hydroxylation of Pro & &
\end{tabular}

crosslinking experiments carried out in the present study under atmospheric conditions were not successful. The reaction time took longer than $7 \mathrm{~d}$, which makes it a nonsustainable process. The $\mathrm{pH}$ of the reaction media is an important parameter, which greatly influences the reaction mechanisms of genipin crosslinking. Not only the reaction pathways are different but also it has been reported that the reaction is faster under acidic conditions. ${ }^{[23,46]}$ In this sense, a different approached using a dense carbon dioxide atmosphere was tested in the present study. Carbon dioxide when soluble in water acidifies the solutions, decreasing the $\mathrm{pH}$ up to 3 . In these conditions, different experiments were carried out in order to evaluate the feasibility of collagen scaffolds with genipin under dense $\mathrm{CO}_{2}$ atmosphere.

The optimization of the process required the study of different processing conditions, namely collagen scaffolds concentration, genipin concentration in solution, and crosslinking time. Samples were processed at $\approx 54$ bar and room temperature as previous works have demonstrated the possibility to crosslink the structures under these conditions. ${ }^{[2]}$ Table 2 describes in more detail the different experimental conditions tested.

Following this approach, the crosslinking reaction time decreased from $>7 \mathrm{~d}$ up to $16 \mathrm{~h}$. The mechanisms behind these observations are still not completely unveiled, nonetheless it has been described that there are two different crosslinking mechanisms depending on the $\mathrm{pH}$ of the reaction media. Under acidic conditions, a nucleophilic attack to an amino group of the polysaccharide occurs, with a consequent opening of the dihydropyran ring of the genipin molecule and a tertiary amine is formed. On the other hand, under basic conditions, the ring-opening reaction of the genipin molecule is derived from a nucleophilic attack by the hydroxyl groups in solution. In this case, aldehyde intermediates are formed, which subsequently suffer aldol condensation. The terminal groups of genipin suffer a Schiff reaction with the amino groups of the polysaccharide forming a cross-linked network. ${ }^{[23,46,47]}$

The success of the crosslinking reaction with genipin is characterized by the appearance of a light blue color in the materials. The experiments carried out indicate that prescaffolds prepared from a concentrated solution of collagen
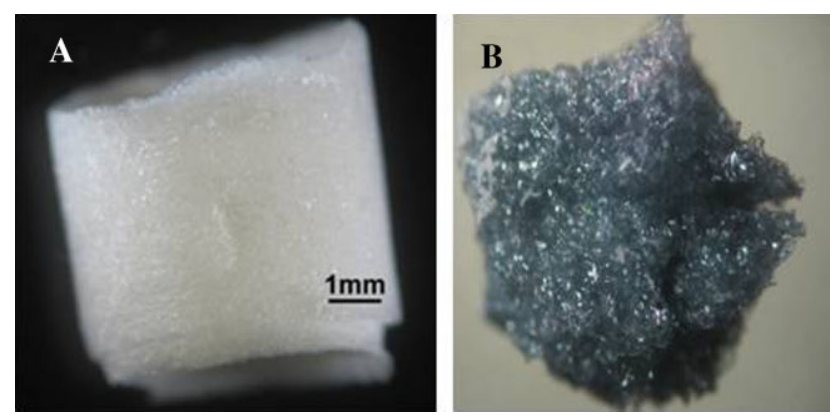

Figure 2. Collagen pre-scaffold (A) and collagen scaffold crosslinked with 0.1 wt\% genipin (B), room temperature and 54 bar for $16 \mathrm{~h}$.
Table 2. Optimization of collagen crosslinking process: Different conditions tested under high pressure $\mathrm{CO}_{2}$.

\begin{tabular}{lcccc}
\hline \multirow{2}{*}{$\begin{array}{l}\text { Collagen } \\
\text { pre-scaffold } \\
{[\%]}\end{array}$} & $\begin{array}{c}\text { Genipin } \\
\text { concentration } \\
{[\%]}\end{array}$ & \multicolumn{3}{c}{ Crosslinking time [h] } \\
\cline { 3 - 5 } & 0.5 & - & - & - \\
\hline 0.5 & 0.3 & - & - & - \\
1 & 0.5 & - & - & $\sqrt{ }$ \\
& 0.1 & - & - & $\sqrt{ }$ \\
2 & 0.3 & - & - & $\sqrt{ }$ \\
& 0.5 & - & $\sqrt{ }$ & $\sqrt{ }$ \\
& & & & \\
$10.1002 / m a b i .201300228$ & & & &
\end{tabular}


react faster than others, which is possibly due to the fact that more functional groups are available for the reaction to take place. Furthermore, in these conditions, it is possible to crosslink the structures with genipin concentrations as low as $0.1 \mathrm{wt} \%$. Figure 2 shows a pre-scaffold $2 \mathrm{wt} \%$ collagen of shark skin (S. canicula), formed by freeze-drying (Figure 2A) and a collagen scaffold crosslinked with $0.1 \mathrm{wt} \%$ genipin overnight $(16 \mathrm{~h})$ at room temperature, in which the crosslinking was promoted by dense $\mathrm{CO}_{2}$ (Figure 2B).

Crosslinking under high pressure carbon dioxide lead additionally to hydrogel foaming, which created an open structure with larger pores, essential for the cell growth and proliferation as well as for nutrients transport and oxygen diffusion into the bulk of the matrices. The water present in the genipin solution is responsible for the behavior observed. Crosslinking experiments carried out with genipin dissolved in pure acetone were not successful and did not promote the foaming of the structure. The mechanism of hydrogel foaming was described by Tsioptsias. ${ }^{[48]}$ Briefly, when the polymer is exposed to carbon dioxide, the water present will dissolve the gas causing the swelling of the polymer. Upon depressurization nucleation occurs both at the water/polymer interface as well as in the water phase itself and the pores are formed. At the same time the structure is stabilized by the Joule-Thomson effect. After complete depressurization the stabilization of the structure produced is achieved by freezing and further freeze-drying of the matrices created. Different acetone: water ratios were tested and from the experiments performed we concluded that the best conditions for the crosslinking reaction were obtained with a ratio 90:10 acetone:water. The necessary presence of water is related with its ability to dissolve carbon dioxide and in this manner leading to the foaming of the amorphous polymer.

\subsection{Mechanical Properties}

The mechanical properties of the scaffolds prepared was evaluated on compression mode on the pre-scaffolds 1 wt\% and on the scaffolds cross-linked with $0.5 \mathrm{wt} \%$ genipin overnight. Figure 3 shows a representative stress/strain curve of the scaffolds.

The differences observed for the prescaffolds and the crosslinked scaffolds demonstrate the differences in the elasticity of the samples, which is also due to the differences observed in the structures, but also due to the crosslinking effect. The compression modulus of the freeze-dried collagen scaffolds prepared was calculated to be $46.3 \mathrm{kPa}$. Cross-

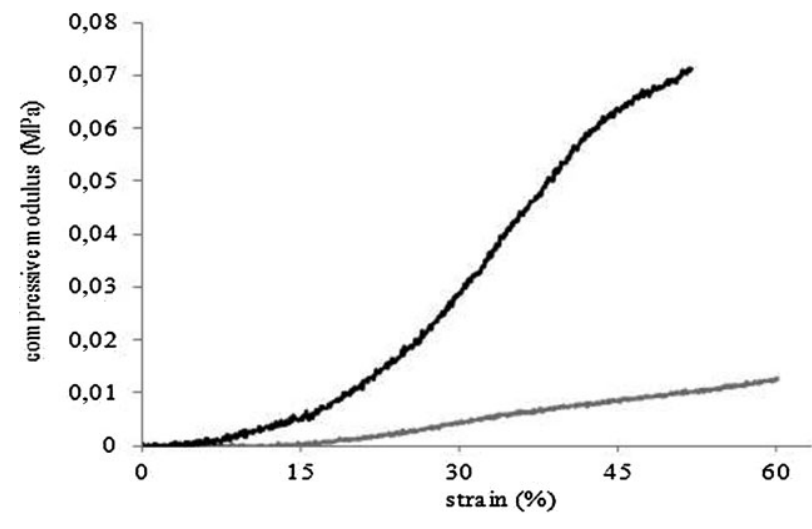

Figure 3. Stress/strain curve of the scaffolds 1 wt\% collagen (gray line) and crosslinked with genipin ( 0.5 wt\%, overnight, black line).

linking of the structure with $0.5 \mathrm{wt} \%$ genipin, overnight lead to an increase of the modulus to $57.3 \mathrm{kPa}$ for the prescaffolds 1 wt\%.

Although the mechanical properties are dependent on the nature of the polymers, crosslinkers and operating conditions used for processing, the results obtained in this work are in good agreement with similar scaffolds produced by the same technique in other works reported in the literature, particularly the case of chitosan scaffolds crosslinked with genipin $(73.9 \pm 7 \mathrm{kPa}) .{ }^{[27]}$

\subsection{Crosslinking Degree}

The structures obtained were characterized by FTIR spectroscopy in total reflection (ATR) mode in order to understand the chemical modifications that took place during the crosslinking (Figure 4). Results showed that a 
higher crosslinking, i.e. a higher genipin concentration is translated by an increase in the intensity of the peaks 1645 and $1506 \mathrm{~cm}^{-1}$, which correspond to the amide I $(C=O)$ and amide II (N-H) bands, respectively. Crosslinked scaffolds also exhibit a weak peak at $1730 \mathrm{~cm}^{-1}$. This peak is attributed to an ester group of genipin ( $\mathrm{C}-\mathrm{O}-\mathrm{O})$ and its decrease indicates the success of the crosslinking. Due to the increase in the genipin concentration form 0.1 to $0.5 \mathrm{wt} \%$ a peak, at $3563 \mathrm{~cm}^{-1}$, attributed to the amine $\left(-\mathrm{NH}_{2}\right)$ appeared became more intense with increasing concentration.

The crosslinking degree was determined for the collagen scaffolds 2 wt\%, 0.1 wt\% genipin, crosslinked overnight as these are the ones that present better properties for further studies. The scaffolds prepared from 2 wt\% collagen and crosslinked with 0.1 wt\% genipin were less fragile and presented higher stability as well as higher mechanical properties. Furthermore, due to the potential cytotoxicity associated with unreacted monomers, which may be impregnated in the structure after processing, the use of a low concentration of crosslinking agent is crucial for a successful in vitro biological performance.

The degree of crosslinking was determined after the ninhydrin assay, which allows the calculation of the percentage of free amino groups remaining in the collagen scaffolds after crosslinking, in comparison with the free amino groups present on the pre-scaffold, according to

Degree of crosslinking $=$

$\underline{(\mathrm{NH} \text { reactive amine })_{\text {fresh }}-(\mathrm{NH} \text { reactive amine })_{\text {fixed }}} \times 100$ $(\mathrm{NH} \text { reactive amine })_{\text {fresh }}$

where fresh and fixed are related to the mole fractions of free $\mathrm{NH}_{2}$ in the non-crosslinked and crosslinked samples, respectively. The results obtained indicate a degree of crosslinking of $25 \%$.

\subsection{Morphological Characterization}

The morphological characterization of the structures is one of the most important parameters in which concerns materials for tissue regeneration. Adequate porosity, mean pore size, and interconnectivity are the main requirements to assess whether a scaffold holds potential to be used for a particular application or not. The cross-section of the crosslinked structures were analyzed by scanning electron microscopy (SEM). Figure 5 represents the microscopic images of the freeze-dried collagen scaffold and the crosslinked collagen scaffold.

The freeze-dried collagen scaffold presents already an adequate morphology for tissue engineering and regenerative medicine. Their highly porous structure coupled with a mean pore size around $50 \mu \mathrm{m}$ and high interconnectivity would be suitable for cell growth, despite the fact that they are not stable when hydrated. After processing with dense carbon dioxide, the crosslinked structure suffers a second foaming stage, which increases the porosity and the interconnectivity of the pores. From the images obtained by microscopy, it is noticeable that the differences in pore size, which are in this case close to $100 \mu \mathrm{m}$, almost the double in comparison to the freeze-dried scaffold.

Micro-CT allows a quantitative evaluation of the common morphological parameters that characterize a 3D structure, such as pore size, porosity and interconnectivity of the structures, as well as the $3 \mathrm{D}$ reconstruction of the matrices prepared. In Figure 6, the 3D reconstruction of the scaffold is presented and the morphometric characterization of the scaffolds is highlighted.

Results show a unimodal pore size distribution with an average pore size of $98 \mu \mathrm{m}$. The structures present $79 \%$ porosity and $76 \%$ interconnectivity, which indicate a strong possibility for the good penetration and cellular proliferation into the scaffold, as well as nutrients and oxygen diffusion into the bulk of the matrix. The values of porosity and interconnectivity of the crosslinked scaffold are
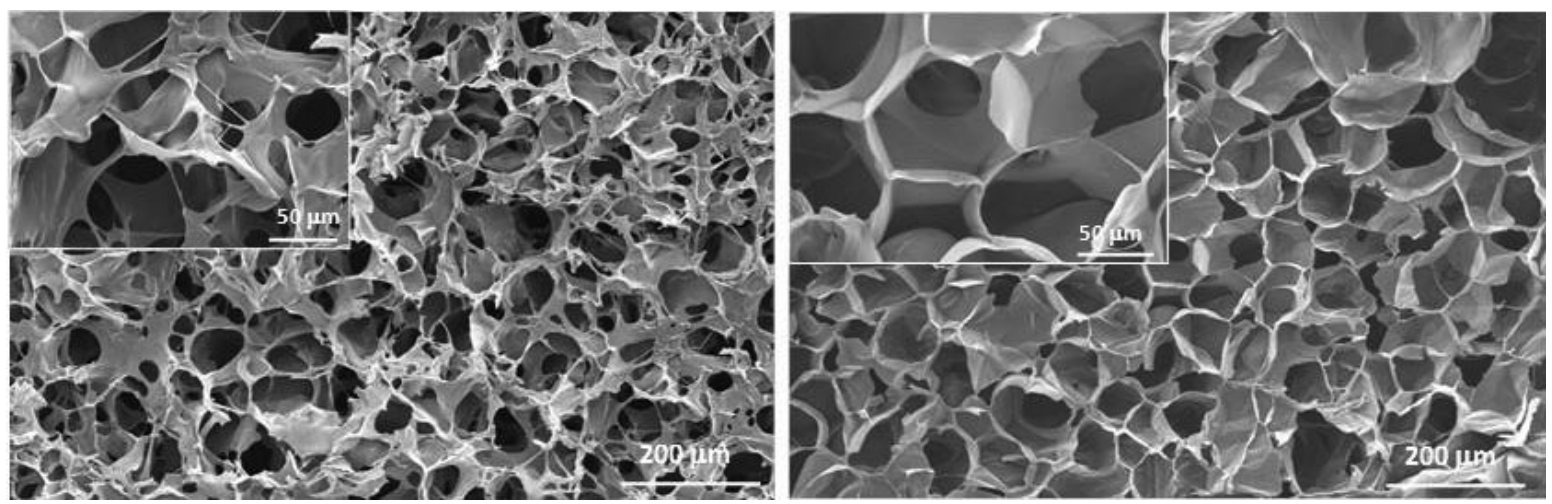

Figure 5. Cross-section by SEM of the freeze-dried collagen scaffold (A) and crosslinked collagen scaffold (B).

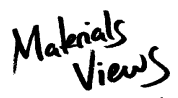

www.MaterialsViews.com
Macromol. Biosci. 2013, DOI: 10.1002/mabi.201300228

(C) 2013 WILEY-VCH Verlag GmbH \& Co. KGaA, Weinheim

Early View Publication; these are NOT the final page numbers, use DOI for citation !! 

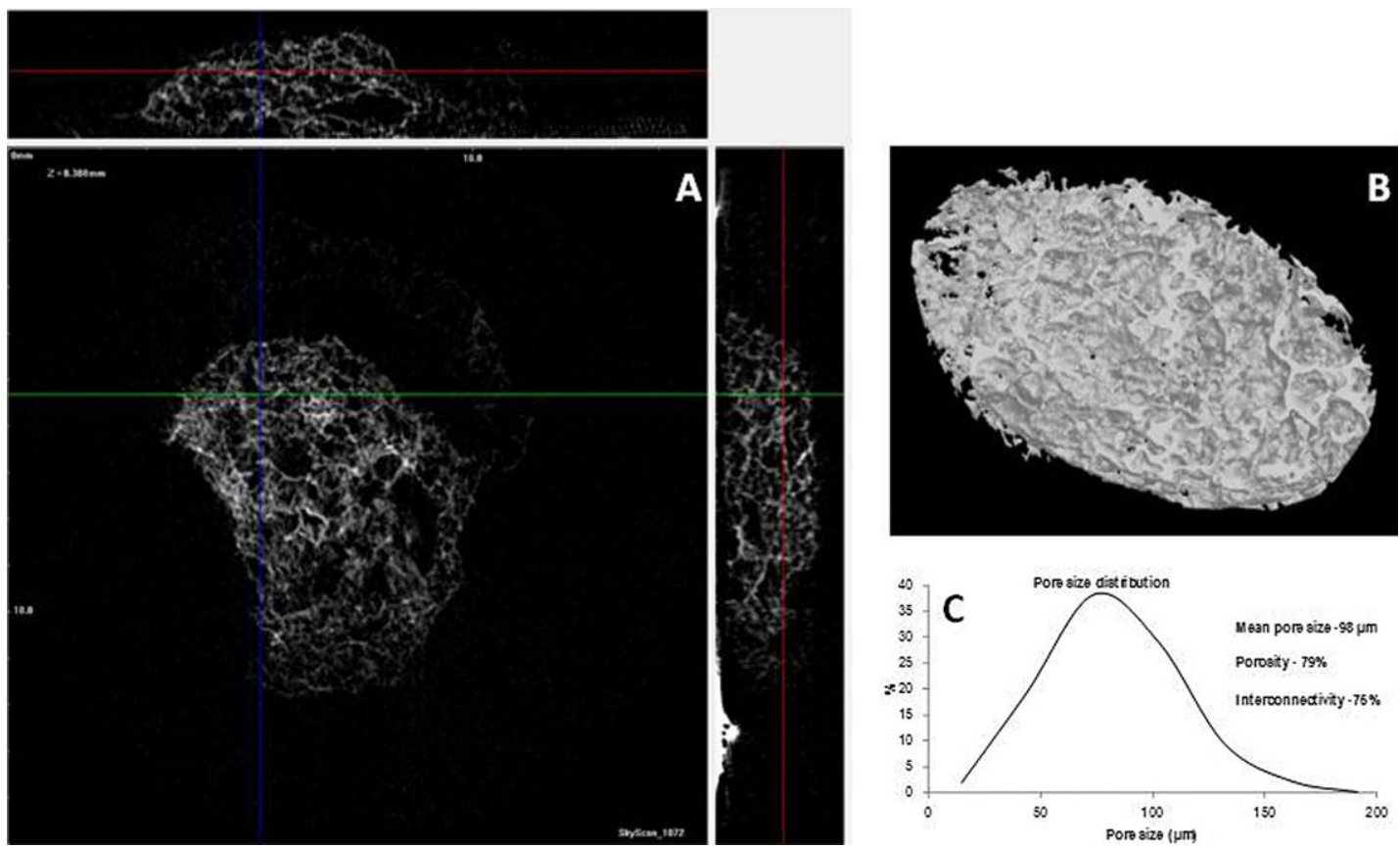

Figure 6. Micro-CT morphological analysis of the 2 wt\% collagen scaffolds crosslinked with o.1 wt\% genipin, overnight: (A) $2 \mathrm{D}$ cross-sections, (B) $3 \mathrm{D}$ reconstruction of the matrix, and (C) morphological characterization of the structures.

significantly higher than the ones of the pre-scaffold, which were determined to be $45 \%$ porosity and $45 \%$ interconnectivity. This increase is due to the second foaming that the material experiences after the crosslinking under dense carbon dioxide atmosphere and depressurization of the system. The morphological parameters were determined in the dry state, which may potentially be different from their characteristics in the wet state. The differences that could be observed do not, however compromise the applications suggested.

\subsection{In vitro Biological Performance}

The stability of the structure was evaluated by the determination of the swelling degree of the structures in PBS and in cell culture media, particularly in DMEM, as the presence of proteins may have a role on the swelling ability and stability of the structures. The swelling was determined as a function of the amount of fluid uptake, by measuring the differences between initial dry weight and wet weight of the samples, after predetermined periods of time. Figure 7 presents the fluid uptake percentage of the collagen scaffolds crosslinked with genipin in the two different immersion media (DMEM and PBS).

The fluid uptake ability is higher in DMEM culture media than in PBS however, the structures are not as stable and after $7 \mathrm{~d}$ the matrices start to lose structure and integrity and eventually degrade. The presence of some proteins may influence the degradation process and may be the justification for the observed behavior. These observations are particularly important for further in vitro cytotoxicity studies, as it is of great importance to stabilize the structure and to maintain it for a longer period of time. The findings of our work suggest that the immersion of the collagen scaffolds in PBS before immersion in culture media stabilizes the structure leading to slower degradation rate.

In vitro cell seeding studies were carried out with 3D matrices prepared from pre-scaffolds of $2 \mathrm{wt} \%$ collagen, with a crosslinking reaction of $16 \mathrm{~h}$. Under these operating

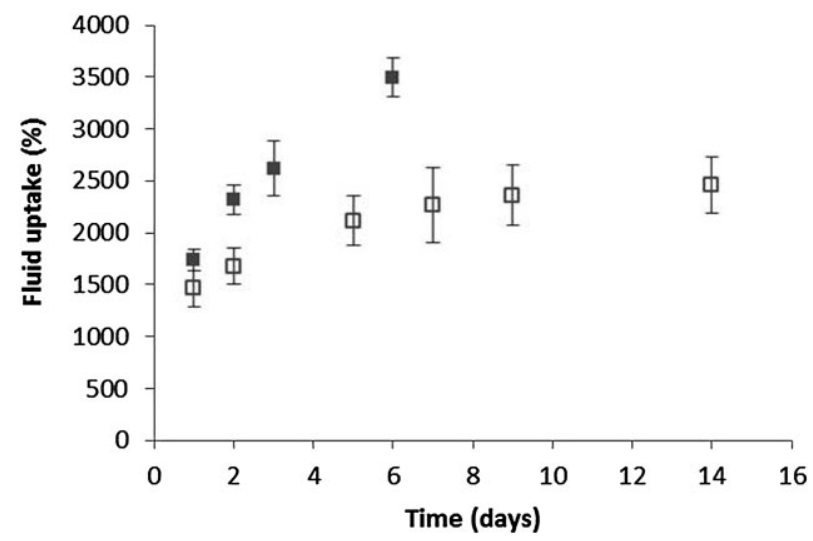

Figure 7. Fluid uptake behavior of the collagen scaffolds crosslinked with genipin in PBS (open squares) and DMEM (filled squares) at $37^{\circ} \mathrm{C}, 60 \mathrm{rpm}$. 

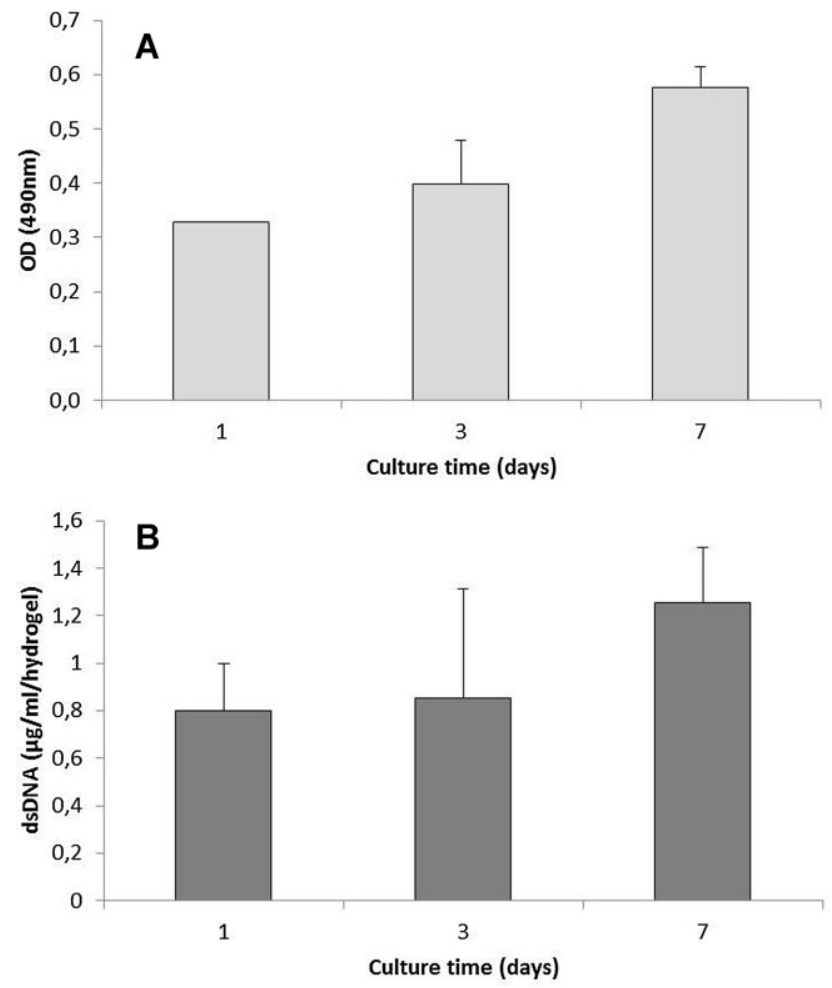

Figure 8. Cell viability (A, MTS assay) and cell proliferation (B, DNA quantification) results after 1,3 , and 7 of cell culture.

conditions, it is possible to use genipin concentrations as low as $0.1 \mathrm{wt} \%$, which although the low cytotoxicity of genipin is favorable for the development of products for biomedical applications. It is, nonetheless, crucial to evaluate the toxicity of the materials prepared prior to move forward into in vivo studies.

Direct contact assays were performed with chondrocytes (ATDC5) as the scaffolds present interesting features for cartilage tissue regeneration. The potential cytotoxicity of the collagen porous structures was evaluated using a standard MTS assay. In this assay, the viability of the cells when in contact with the material is evaluated, at the same time the effect of any potential toxic leachable from the construct is also determined. Cell growth and proliferation was determined by DNA quantification. Figure 8 presents the MTS and DNA results.

The MTS results demonstrate that the cells seeded on the crosslinked collagen matrices remain viable within the culture time studied. Cells are able to grow on the 3D scaffolds, which is also corroborated by the DNA results that show a slow increase in cell proliferation. Although no statistically significant differences were observed between the three time points, it is possible to conclude that the scaffolds are non-cytotoxic.

Cell morphology and adherence was evaluated on crosslinked collagen scaffolds, after cell seeding, by SEM and by fluorescent microscopy (Figure 9). SEM micrographs show in day 1 cells with a round shape indicating that they still did not adhere to the surface, however after $3 \mathrm{~d}$ cells have adhered to the surface and present their characteristic morphology. After $7 \mathrm{~d}$ in culture, the surface of the scaffold is covered with cells that start to colonize the bulk of the scaffold. The results observed by SEM are consistent with the Calcein-AM/DAPI analysis, suggesting that ATDC5 are able to adhere to the surface and proliferate.


Figure 9. SEM micrographs (A-C) and fluorescent microscopy images (calcein/DAPI) (D-F) of ATDC5 cultured in hydrogels for $1 \mathrm{~d}(\mathrm{~A}$ and $\mathrm{D}), 3$ $\mathrm{d}(\mathrm{B}$ and $\mathrm{E})$, and $7 \mathrm{~d}(\mathrm{C}$ and $\mathrm{F})$.

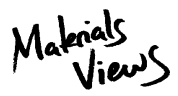

www.MaterialsViews.com
Macromol. Biosci. 2013, DOI: 10.1002/mabi.201300228

(C) 2013 WILEY-VCH Verlag GmbH \& Co. KGaA, Weinheim

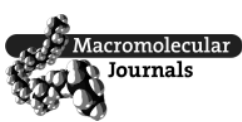

Early View Publication; these are NOT the final page numbers, use DOI for citation !! 


\section{Conclusion}

The development of crosslinked three-dimensional hydrogels of marine collagen extracted from shark skin was carried out under dense carbon dioxide atmosphere. The optimization of process conditions demonstrated that successful crosslinking with genipin under dense $\mathrm{CO}_{2}$ atmosphere could be achieved at room temperature, 55 bar and $16 \mathrm{~h}$. The structures were crosslinked with a low genipin concentration of $0.1 \mathrm{wt} \%$, which may present additional advantages, especially for tissue engineering and regenerative medicine applications, as the toxicity of the material is not compromised. In comparison with conventional crosslinking reaction, the processing time decrease significantly as the reaction under atmospheric conditions was only achieved after $7 \mathrm{~d}$. The obtained scaffolds present a highly porous and interconnected structure allowing cell adhesion, growth, and proliferation. In this work, we report for the first time the development of 3D architectures of collagen from shark skin. The results obtained demonstrate the potential of marine collagen structures to be used as scaffolds for cartilage tissue regeneration.

Acknowledgements: The research leading to these results has received funding from the European Union Seventh Framework Programme (FP7/2007-2013) under grant agreement no. KBBE2010-266033 (project SPECIAL) and from FEDER through POCTEP Project 0330 IBEROMARE 1 P. Portuguese Foundation for Science and Technology is also gratefully acknowledged for post-doc grants of J. Moreira-Silva and T.H. Silva.

Received: May 8, 2013; Revised: June 26, 2013; Published online: DOI: $10.1002 / \mathrm{mabi} .201300228$

Keywords: collagen; hydrogels; marine biotechnology; supercritical fluids; tissue engineering

[1] J. F. Mano, G. A. Silva, H. S. Azevedo, P. B. Malafaya, R. A. Sousa, S. S. Silva, L. F. Boesel, J. M. Oliveira, T. C. Santos, A. P. Marques, N. M. Neves, R. L. Reis, J. R. Soc. Interface 2007, 4, 999.

[2] C. H. Lee, A. Singla, Y. Lee, Int. J. Pharm. 2001, 221, 1.

[3] C. Englert, T. Blunk, R. Muller, S. von Glasser, J. Baumer, J. Fierlbeck, I. Heid, M. Nerlich, J. Hammer, Arthritis Res. Ther. 2007, 9, R47.

[4] W. Garnjanagoonchorn, L. Wongekalak, A. Engkagul, Chem. Eng. Process. Process Intensification 2007, 46, 465.

[5] I. Kolodziejska, Z. E. Sikorski, C. Niecikowska, Food Chem. 1999, 66, 153.

[6] M. C. Gomez-Guillen, J. Turnay, M. D. Fernandez-Diaz, N. Ulmo, M. A. Lizarbe, P. Montero, Food Hydrocolloids 2002, 16, 25.

[7] S.-K. Kim, E. Mendis, Food Res. Int. 2006, 39, 383.

[8] J. Morales, P. Montero, A. Moral, J. Agric. Food Chem. 2000, 48, 2142.
[9] N. Takeshi, N. Suzuki, Food Chem. 2000, 68, 277.

[10] D. Swatschek, W. Schatton, J. Kellermann, W. E. Müller, J. Kreuter, Eur. J. Pharm. Biopharm. 2002, 53, 107.

[11] T. H. Silva, A. R. C. Duarte, J. Moreira-Silva, J. F. Mano, R. L. Reis, "Biomaterials from Marine-Origin Biopolymers," in: Biomimetic Approaches for Biomaterials Development, J. F. Mano, (Ed., Wiley-VCH, Weinheim 2012.

[12] T. H. Silva, A. Alves, B. M. Ferreira, J. M. Oliveira, L. L. Reys, R. J. F. Ferreira, R. A. Sousa, S. S. Silva, J. F. Mano, R. L. Reis, Int. Mater. Rev. 2012, 57, 276.

[13] R. Pallela, J. Venkatesan, V. R. Janapala, S. K. Kim, J. Biomed. Mater. Res. A 2012, 100A, 486.

[14] T. Nagai, T. Ogawa, T. Nakamura, T. Ito, H. Nakagawa, K. Fujiki, M. Nakao, T. Yano, J. Sci. Food Agric. 1999, 79, 855.

[15] F. Pati, P. Datta, B. Adhikari, S. Dhara, K. Ghosh, P. K. Das Mohapatra, J. Biomed. Mater. Res. A 2010, 100A, 1068.

[16] R. Duan, J. J. Zhang, X. O. Du, X. C. Yao, K. Konno, Food Chem. 2009, 112, 702.

[17] P. Kittiphattanabawon, S. Benjakul, W. Visessanguan, F. Shahidi, LWT Food Sci. Technol. 2010, 43, 792.

[18] Y. Takagi, K. Ura, J. Nanosci. Nanotechnol. 2007, 7, 757.

[19] P. Singh, S. Benjakul, S. Maqsood, H. Kishimura, Food Chem. 124, 97.

[20] E. Song, S. Yeon Kim, T. Chun, H.-J. Byun, Y. M. Lee, Biomaterials 2006, 27, 2951.

[21] D. Leary, M. Vierros, G. L. Hamon, S. Arico, C. Monagle, Marine Policy 2009, 33, 183.

[22] D. M. Veríssimo, R. F. C. Leitão, R. A. Ribeiro, S. D. Figueira, A. S. B. Sombra, J. C. Gães, G. A. C. Brito, Acta Biomater. 2010, 6, 4011.

[23] R. A. A. Muzzarelli, Carbohydr. Polym. 2009, 77, 1.

[24] S. S. Silva, A. Motta, M. T. Rodrigues, A. F. M. Pinheiro, M. E. Gomes, J. F. Mano, R. L. Reis, C. Migliaresi, Biomacromolecules 2008, 9, 2764

[25] Y. Yuan, B. M. Chesnutt, G. Utturkar, W. O. Haggard, Y. Yang, J. L. Ong, J. D. Bumgardner, Carbohydr. Polym. 2007, 68, 561.

[26] L.-P. Yan, Y.-J. Wang, L. Ren, G. Wu, S. G. Caridade, J.-B. Fan, L.-Y. Wang, P.-H. Ji, J. M. Oliveira, J. T. Oliveira, J. F. Mano, R. L. Reis, J. Biomed. Mater. Res. A 2010, 95A, 465.

[27] C. Ji, N. Annabi, A. Khademhosseini, F. Dehghani, Acta Biomater. 2011, 7, 1653

[28] S.-M. Lien, L.-Y. Ko, T.-J. Huang, Mater. Sci. Eng. C 2010, 30, 631.

[29] N. Annabi, S. M. Mithieux, E. A. Boughton, A. J. Ruys, A. S. Weiss, F. Dehghani, Biomaterials 2009, 30, 4550.

[30] N. Annabi, S. M. Mithieux, A. S. Weiss, F. Dehghani, Biomaterials 2010, 31, 1655.

[31] N. Annabi, S. M. Mithieux, A. S. Weiss, F. Dehghani, Biomaterials 2009, 30, 1.

[32] C. Ji, A. Khademhosseini, F. Dehghani, Biomaterials 2011, 32, 9719.

[33] F. Dehghani, N. Annabi, Curr. Opin. Biotechnol. 2011, 22, 661.

[34] S. M. Lien, W. T. Li, T. J. Huang, Mat. Sci. Eng. C 2008, 28, 36.

[35] F. J. Chung, M. Sugimoto, J. L. Koh, G. A. Ameer, Tissue Eng. C 2012, 18, 113

[36] A. R. C. Duarte, J. F. Mano, R. L. Reis, J. Bioactive Compat. Polym. 2009, 24, 385

[37] A. R. C. Duarte, J. F. Mano, R. L. Reis, Int. Mater. Rev. 2009, 54, 214.

[38] U. K. Laemmli, Nature 1970, 227, 680.

[39] A. A. Karim, R. Bhat, Food Hydrocolloids 2009, 23, 563

[40] M. C. Gomez-Guillen, B. Gimenez, M. E. Lopez-Caballero, M. P. Montero, Food Hydrocolloids 2011, 25, 1813. 
[41] C. G. Sotelo, M. B. Comesaña, P. R. Ariza, R. I. Pérez-Martín, J. Aquat. Food Prod. Technol. 2013.

[42] D. R. Stamov, T. Pompe, Soft Matter 2012, 8, 10200.

[43] J. C. Horng, F. W. Kotch, R. T. Raines, Protein Sci. 2007, 16, 208.

[44] A. Jongjareonrak, S. Rawdkuen, M. Chaijan, S. Benjakul, K. Osako, M. Tanaka, LWT Food Sci. Technol. 2010, 43, 161.

[45] J. M. Regenstein, P. Zhou, "Collagen and Gelatin from Marine By-Products". in: Maximising the Value of Marine By-Products, F. Shahidi, (Ed., Woodhead Publishing Lt:
Memorial University of Newfoundland, St. Johns, Canada 2006, pp. 279-303.

[46] M. F. Butler, Y. F. Ng, P. D. A. Pudney, J. Polym. Sci. Part A: Polym. Chem. 2003, 41, 3941.

[47] F. L. Mi, S. S. Shyu, C. K. Peng, J. Polym. Sci. Part A: Polym. Chem. 2005, 43, 1985.

[48] C. Tsioptsias, M. K. Paraskevopoulos, D. Christofilos, R. Andrieux, C. Panayiotou, Polymer 52, 2819.

[49] M. Zhang, W. T. Liu, G. Y. Li, Food Chem. 2009, 115, 826.

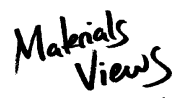

www.MaterialsViews.com
Macromol. Biosci. 2013, DOI: 10.1002/mabi.201300228

(C) 2013 WILEY-VCH Verlag GmbH \& Co. KGaA, Weinheim

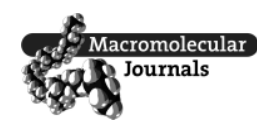

11

Early View Publication; these are NOT the final page numbers, use DOI for citation !! 7 